\title{
An Approach for More Efficient Energy Consumption Based on Real-Time Situational Awareness
}

\author{
Yongchun Xu, Nenad Stojanovic, Ljiljana Stojanovic, \\ Darko Anicic, and Rudi Studer \\ FZI Research Center for Information Technology \\ 76131 Karlsruhe, Germany \\ name. familyname@fzi.de
}

\begin{abstract}
In this paper we present a novel approach for achieving energy efficiency in public buildings (especially sensor-enabled offices) based on the application of intelligent complex event processing and semantic technologies. In the nutshell of the approach is an efficient method for realizing the real-time situational awareness that helps in recognizing the situations where a more efficient energy consumption is possible and reaction on those opportunities promptly. Semantics allows a proper contextualization of the sensor data (i.e. its abstract interpretation), whereas complex event processing enables the efficient real-time processing of sensor data and its logic-based nature supports a declarative definition of the situations of interests. The approach has been implemented in the iCEP framework for intelligent Complex Event Reasoning. The results from a preliminary evaluation study are very promising: the approach enables a very precise real-time detection of the office occupancy situations that limit the operation of the lighting system based on the actual use of the space.
\end{abstract}

Keywords: Energy Efficiency, Complex Event Processing, Semantic Technology, Office Occupancy Control.

\section{Introduction}

Real-time processing has become very important for sensor-based applications, since the quantity of data being generated from sensors requires on-the-fly processing and immediate reaction in order to be effective. There are many examples, starting from item-tracking in RFID-supported logistics to remote patient monitoring in eHealth. Indeed, real-time awareness enables the detection of problems (e.g. a damaged item in a delivery, or an acute health problem in a patient) as soon as they happen, so that the reaction can be successfully performed. Note that the same mechanism can be used for preventive reactions, i.e. reacting before a problem would happen.

In the nutshell of this mechanism is the ability to recognize in real-time ${ }^{1}$ (or even ahead of time) some interesting situations, what is called "real-time situational awareness". Note that this goes beyond the traditional (static) situational awareness

${ }^{1}$ We consider "business real-time" as the criteria for declaring something to be processed in real-time. 
(like in [1]) that is focused on the understanding a situation (if possible in realtime). Real-time situational awareness introduces the notion of real-time emergency: the main goal is to recognize a situation of interest as soon as possible in order to be able to react on it properly.

On the other hand, such a process introduces several challenges for the processing of sensor-data:

a) it should be very efficient in order to retain its "real-time" flavor and

b) it should be very flexible in order to deal with various and dynamically changing patterns (situations) of interests (that should be recognized in real-time).

Complex event processing is a technology that can resolve these challenges.

Energy efficiency is one of application areas, where real-time situational awareness can bring a substantial added value. Smart Grid is a well-known example: Smart meters $^{2}$ enable real-time publishing of information about energy consumption of a user (usually once every seven minutes), which consequently can support the realtime optimization of the energy production. Decreasing energy consumption in buildings, public spaces and households is another very promising area for applying real-time situational awareness. It has been shown that the creation of the awareness about the current (in real-time) energy consumption in a household can bring itself up to $20 \%$ savings in electricity bills. Beside these passive savings, active energy savings by switching off electrical devices in particular situations are very common methods in reducing energy consumption ${ }^{3}$.

Although well developed, current approaches for achieving energy efficiency seem to be "inefficient": they are usually dealing with customized solutions tuned to the predefined energy consumption scenarios. The consequence is that the costs for introducing and maintaining these solutions are quite high, since e.g. each new scenario (the so-called energy consumption pattern) has to be modeled separately. On the other hand, due to a high variation in the energy consumption profile (e.g. the spatial distribution of energy consumers), energy consumption patterns are very variable and modeling new patterns is more a rule than an exception. Therefore, although oriented towards real-time recognition of the interesting situations, these approaches are suffering from the inflexibility in the detection process, e.g. by not having available a declarative description of the situations to be detected and by not performing an intelligent processing (reasoning) on the incoming data. Obviously, the application of semantic technologies can be very promising for resolving these challenges.

In this paper we present a novel approach for achieving energy efficiency that exploits real-time situational awareness based on the use of Complex Event Processing and Semantic Technologies. The main idea is to enable a semantic-based description of the situations of interests (i.e. energy consumption patterns) and perform reasoning about those situations in real-time. The approach leverages on our work in the domain of intelligent Complex Event Processing (iCEP) ${ }^{4}$, especially complex event reasoning, that combines a very efficient in-memory processing (on the fly) of a huge amount of streaming data and the reasoning (on the fly) using available domain knowledge.

\footnotetext{
${ }^{2}$ http://www.smartmeters.com/faqs.html

${ }^{3} \mathrm{http}: / / \mathrm{www}$.greentrac.com/

${ }^{4}$ See iCEP.fzi.de
} 
The approach has been implemented using the iCEP framework and deployed in our experimental environment that supports testing novel technologies with a higher users' involvement. We have performed a case study related to the office occupancy control that limits the operation of the lighting system based on the actual use of the space. Preliminary evaluation tests have shown very promising results regarding the usability and the efficiency of the approach: the approach is able to abstract from particular patterns to be recognized into general/declarative situations to be reasoned about.

The paper is structured in the following way:

In the second section we give more details about our Energy Efficiency use case, from the real-time consumption point of view. In the third section we outline the architecture of our solution. In section four we describe some evaluation details, whereas section five elaborates briefly on the related work. In section six we give some concluding remarks.

\section{Energy Efficiency in Offices: State of the Art and Requirements Analysis}

There are several studies which show that up to $70 \%$ of the energy consumption in an office environment can be saved just by "creating awareness" about the current energy consumption. This is especially related to the very low electricity consumption of the equipment and lighting. For example, while according to Logicalis 5 , 94\% of workers surveyed turn their lights off at home, only $66 \%$ thought about doing the same at work. Turning the lights off all too often gets ignored by offices, whose lights continue to shine even after everyone has gone home. On the other hand, many of the energy saving activities can be automated, like "whenever workers leave a room, the lights should be turned off" (see more examples in the Evaluation section).

There are several approaches which are dealing with the automation of this process, which are usually taking into account the following factors for the lighting issues:

- control of the user's presence in an office, as a necessary condition to turn on the light;

- regulation of the artificial light, in relation to the natural light level;

- possibility of a manual regulation of the light, forcing the automatic regulation, in order to better meet the user's needs.

Therefore, the automation of the energy saving process is related to an efficient sensing of the current situation in an office and reacting in particular situations. The best examples are the so-called occupancy controls that limit the operation of the lighting system based on the actual use of the space. Unlike scheduling controls, they do not operate by a pre-established time schedule. Instead, the system senses when the space is occupied and turns the lights on. When the system senses that there has been no activity in the space, it assumes the space is unoccupied and turns the lights off. To

\footnotetext{
${ }^{5}$ http://www.energysavingsecrets.co.uk/HowToRunAnEnergyEfficientOffice.html
} 
prevent the system from turning the lights off while the space is still occupied but there is very little activity, a time delay typically ranging from 1 to 15 minutes can be programmed into the controls.

However, as already mentioned in the Introduction, current approaches are based on a fix set of rules that are designed for a particular scenario. Let's illustrate this on the example depicted in Figure 1: an office with six desks, each equipped with a lamp. The task is to enable switching off the corresponding lamp when a person is leaving the room. The most advanced systems would model several patterns that describe situations that a person is leaving the room which implies the need for switching off the light at her/his desk. A pattern for the region F would be (cf. Figure 1):

If sequence (RegionF, RegionD, RegionB, Door) Then SwitchOff(LampF)

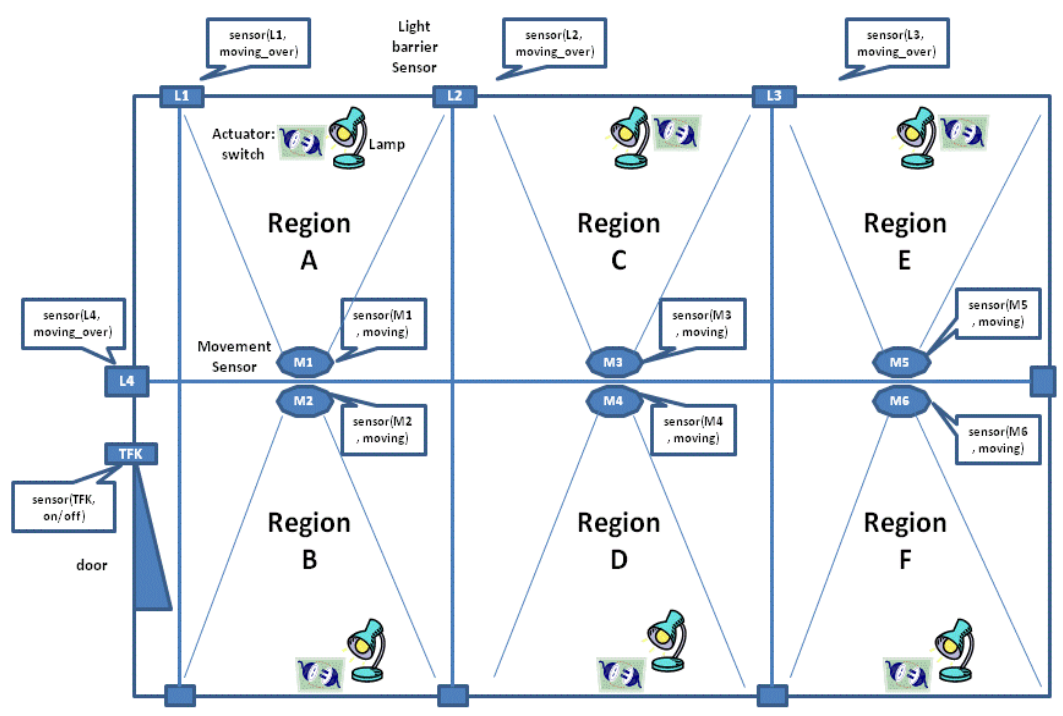

Fig. 1. The distribution of sensors in a smart office. There are three types of sensors: 1) contact sensors (TFK - attached to the door), 2) moving sensors (M1 - M6) and Light barrier sensors (L1-L4)

The main drawbacks of such an approach are:

1) the energy saving patterns are "hard-coded", which means that all patterns must be explicitly defined in order to be taken into account;

2) there is no abstraction in the pattern definition, so that any kind of generalization is excluded;

3) the patterns are "static", so that any kind of changes in the initial setting cannot be realized easily.

Consequently, the requirements for a novel approach are related to:

1) a declarative description of energy saving patterns in order to enable an abstract definition of the situations to be recognized; 
2) the usage of domain knowledge in order to support the use of implicit information in the detection process;

3) the management of patterns, including their evolution.

In the rest of the paper we present such an approach.

\section{3 iCEP Approach for the Energy Efficiency}

In order to resolve the above mentioned problems, we have developed a logic-based approach for the automatic control of the energy consumption in an office scenario. In the nutshell of the approach is a very efficient complex event processing supported by deductive reasoning capabilities, implemented in the ETALIS engine [2]. The data processed by ETALIS are coming from sensors placed in the office (cf. Figure 1). Sensors are semantically described using ontologies. In the rest of this section we present the information model behind the approach and give more details about the underlying processing system.

\subsection{Information Model}

Figure 2 represents the information model used in the proposed approach.

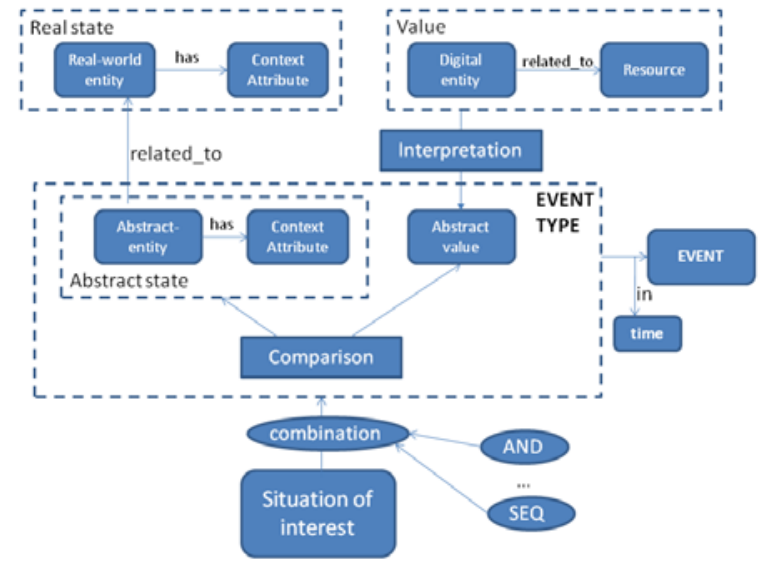

Fig. 2. Information model of the system

It consists of three layers: the raw data provided by sensors, the Digital Entities provided by sensor resources and the context information provided by the advanced system components or context-level resources.

The raw data consists of the value that the sensor provides, e.g., the numerical value 25. A resource may augment this information with meta-information, e.g. that the measured value is a temperature that it is in degrees Celsius that it was measured by sensor $\mathrm{X}$ at a certain point in time etc. We call the resulting information Digital Entity. 
This information is not yet contextualized, i.e., we may know that it is a temperature, but not what this temperature describes, e.g., is this the indoor temperature of a room or the temperature within the fridge? This information is modeled by the context information. The real world is modeled as Real-world entities, which are further described by the context attributes. The Real-world entities have an identifier and an entity type. The entity type, e.g., person, place, or car, etc. defines the context attributes of an entity of interest. For example, a person can have a blood pressure; whereas a car can have a certain fuel level. The context attributes have an attribute name, e.g., "hasIndoorTemperature", an attribute type, e.g., "temperature" and a context value. The context value consists of a Digital Entity plus some quality of information parameters. This quality of information may be different from the quality information that was provided by the observation and measurement, e.g., the accuracy for a room temperature may be calculated as a function of the accuracy of the Digital Entity and the reliability that the temperature sensor provides the temperature of the room.

In order to deal with situations we introduce Abstract Entities that correspond to the abstraction of the Real-world entities in a given application context. For example, a Region in Figure 1 is an Abstract entity. Similarly to a Real-world entity, an Abstract entity is associated to some Context attribute, which has some Abstract value. Note that Abstract value is a matter of the interpretation of the raw data (see Figure 2).

Finally, a situation of interests is a combination of several Abstract states and Values that represent the so-called Event types. Combinations are based on event processing operators (AND, OR, SEQ, etc. [3].

Events represent instantiations of Event types in real time. Those events are processed by ETALIS ${ }^{6}$ engine.

The sensor system takes advantages of using semantic technologies. We use an ontology that is specified in W3C RDF/XML standard format to manage the sensor information and the background domain knowledge (see section 3.4). Our sensor system integrates ETALIS engine, which has the ability to perform stream reasoning with the background knowledge (ontologies) to achieve a high processing performance.

\subsection{ETALIS}

ETALIS Language for Events [2] is a logic-based formalism for Complex Event Processing (CEP) and Stream Reasoning. It uses SWI-Prolog Semantic Web Library ${ }^{7}$ to represent an RDF/XML ontology as a set of Prolog rules and facts. ETALIS is an open-source implementation of the language. The language and a corresponding implementation are based on a novel event processing strategy which detects complex events by maintaining the intermediate states. Every time an atomic event (relevant w.r.t. the set of monitored events) occurs, the system updates the internal state of complex events. Essentially, this internal state encodes what atomic events are still missing for the completion a certain complex event. Complex events are detected as

\footnotetext{
${ }^{6} \mathrm{http}: / /$ code.google.com/p/etalis/

${ }^{7}$ http://www.swi-prolog.org/pldoc/package/semweb.html
} 
soon as the last event required for their detection has occurred. Descriptions telling which occurrence of an event drive the detection of complex events (including the relationships between complex events and events they consist of) are given by deductive rules. Consequently, detection of complex events then amounts to an inference problem.

Event processing formalisms based on deductive or logic rules $[4,5,6]$ have been attracting considerable attention as they feature formal, declarative semantics. Declarative semantics of a CEP system prescribe what the system needs to detect, i.e., a user does not need to worry how that will be detected. In this respect declarative semantics guarantees predictability and repeatability of results produced by a CEP system. Moreover, CEP systems based on deductive rules can process not only events, but also any additional background knowledge relevant with respect to the detection of complex situations in real-time. Hence a rule-based approach enables a high abstraction level and a uniform framework for realizing knowledge-based CEP applications (i.e., specification of complex event patterns, contextual knowledge, and their interaction). Such applications can be further supported by machine learning (more specifically data mining) tools, to automate the construction and refinement of event patterns (see [7]). Although the machine learning support per se is out of scope of this paper, we want to emphasize the importance of the formal, rule-based semantics which can further enable automated construction of both, event patterns and the background knowledge. These features are beyond capabilities of existing approaches $[8,9,10]$, and this is one of reasons why ETALIS follows a logic rulebased approach for event processing.

In the following, we identify a number of benefits of the ETALIS event processing model, realized via deductive rules: First, a rule-based formalism (like the one we present in this paper) is expressive enough and convenient to represent diverse complex event patterns. Second, a formal deductive procedure guarantees the correctness of the entire event processing. Unlike reactive rules (production rules and ECA rules), declarative rules are free of side-effects; the order in which rules are evaluated is irrelevant. Third, although it is outside the scope of this paper, a deductive rule representation of complex events may further help in the verification of complex event patterns defined by a user (e.g., by discovering patterns that can never be detected due to inconsistency problems). Further on, ETALIS can also express responses on complex events, and reason about them in the same formalism [11]. Fourth, by maintaining the state of changes, the ETALIS event model is also capable of handling queries over the entire space (i.e. answering queries that span over multiple ongoing detections of complex events). Ultimately, the proposed event model allows for reasoning over events, their relationships, entire state, and possible contextual knowledge available for a particular domain (application). Reasoning in the ETALIS event model can be further exploited to find ways to reach a given aim, which is a task that requires some intelligence. For example, an application or a service needs to reach a stable or known (desired) state. To achieve this, the system has to have a capability to reason about, or to asses states (in a changing environment). Another example is to just "track and trace" the state of any entity at any time (in order to be able to "sense and respond" in a proactive way).

Technically, ETALIS approach is based on the decomposition of complex event patterns into intermediate patterns (i.e. goals). The status of achieved goals is 
materialized as first class citizens of a fact base. These materialized goals show the progress toward completion of one or more complete event patterns. Such goals are automatically asserted by rules as relevant events occur. They can persist over a period of time "waiting" in order to support detection of a more complex goal or complete pattern. Important characteristics of these goals are that they are asserted only if they are used later on (to support a more complex goal or an event pattern), that goals are all unique and persist as long as they remain relevant (after that they can be deleted). Goals are asserted by rules which are executed in the backward chaining mode. The notable property of these rules is that they are event-driven. Hence, although the rules are executed backwards, overall they exhibit a forward chaining behavior. For more information, an interested reader is referred to [2].

\subsection{Example}

As already mentioned, one of the main advantages of our approach is the possibility to define the situations of interests in a declarative way and reason about them based on the incoming sensor data.

In order to illustrate the abstractions introduced by ETALIS, we present here a very illustrative example for the occupancy control based on the office context presented in Figure 1.

In the traditional approaches, the situation of interests:

"a person left the room and her/his desk lamp should be switched off within 5 sec" must be described by using one rule for each possible situation. An example is illustrated in Figure 3: a person left the room by traversing from Region F, through Region D and B till the door.

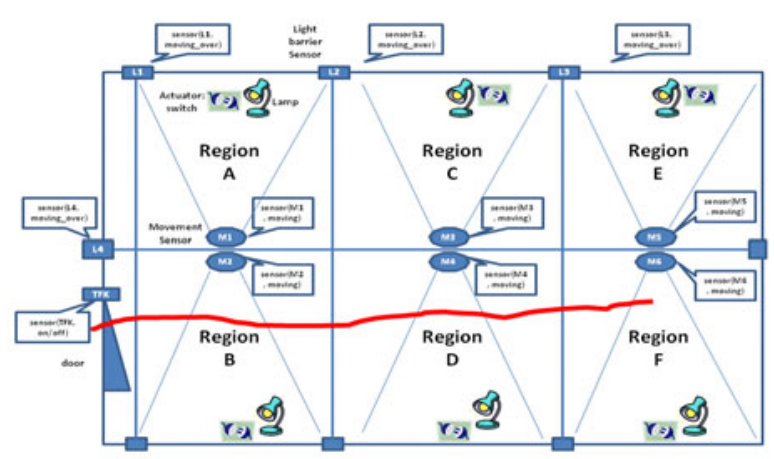

Fig. 3. A possible path from the office desk to the door: a situation that can lead to switching off the lamp at the desk in the Region $\mathrm{F}$

Therefore, traditional approaches must cover use all possible "evacuation" paths which is a tedious and error prone process. The situation is even worse when we consider that the distribution of objects in the office can be changed - the whole set of rules must be rewritten. 
On the other hand, in our approach there is only one logic-based statement that covers all requested situations, by describing them declaratively:

Namespace: cep: http://www.icep.fzi.de/cepsensor.owl\#

Pattern.event:

door_open <- status('cep:door', 'cep:door_opened').

status(A, B) <- sensor(X, Y)

WHERE

(rdfs_individual_of(Sensor, 'cep:Sensor'),

rdf(Sensor, 'cep:hasName', X),

$\operatorname{rdf}($ State, 'cep:hasValue', Y),

rdfs_individual_of(State, 'cep:State'),

$\operatorname{rdf}($ B, 'cep:detectedWithState', State),

rdfs_individual_of(B, 'cep:Status'),

$\operatorname{rdf}($ Sensor, 'cep:locatedIn', A)).

movement(Loc1,Loc2) <- status(Loc1, 'cep:movementInRegion') SEQ status(Bord, 'cep:moveover') SEQ status(Loc2, ‘cep:movementInRegion')

WHERE

(rdfs_individual_of(Loc1, 'cep:Region'),

rdfs_individual_of(Loc2, 'cep:Region'),

rdfs_individual_of(Bord, 'cep:Borderline'),

$\operatorname{rdf(Loc1,~'cep:hasNeighbor',~Loc2),~}$

$\operatorname{rdf(Loc1,~'cep:hasBorderline',~Bord),~}$

$\operatorname{rdf(Loc2,~'cep:hasBorderline',~Bord))2sec.~}$

comment: this statement detects the situation that a person has changed the region, if within 2 sec the movement sensor and light barrier sensor for a Region has been activated

movement(Loc1,Loc3) <- movement(Loc1,Loc2) SEQ movement(Loc2,Loc3) .

movement(Loc, 'cep:door') <- (movement(Loc, 'cep:regionB') SEQ status(Bord, 'cep:moveover')) 2sec.

comment: this statement is the most crucial one: by introducing recursive rules we are able to describe all possible paths which are containing succeeding regions

SwitchOff(Loc) <- (movement(Loc, 'cep:door') SEQ door_open)5sec.

comment: this statement detects the situation that a person has left the room (after a sequence of traversing between regions) and that after 5 sec the light at the starting location should be switched off

Note that the particular state of the world (like this presented in Figure 2) is represented in the domain ontology and the CEP engine (ETALIS) is accessing that knowledge in the real time.

rdf(?Subject, ?Predicate, ?Object)

is a function in SWI-Prolog Semantic Web Library . It is an Elementary query for triples. Subject and Predicate are atoms representing the fully qualified URL of the resource. Object is either an atom representing a resource or literal (Value) if the object is a literal value.

rdfs_individual_of(?Resource, ?Class)

is a function in SWI-Prolog Semantic Web Library. It tests whether the Resource is an individual of class. It returns true if Resource is an individual of Class. This implies Resource has an rdf:type property that refers to Class or a sub-class thereof. It can be used to test, to generate classes Resource belongs to or to generate individuals described by Class. 


\subsection{Domain Ontology}

The structure of the ontology used in our scenario is shown in Figure 4.

The Sensor class hierarchy on the top left models the sensors of the scenario such as contact sensor, movement sensor and light barrier sensor. Each sensor type has one or several states, which describe the physical sensing information of sensors, e.g. the contact sensor has two states: on or off. The states of sensors are modeled by using the class State.

The Actuator class hierarchy on the top right of the Figure 4, similar to the Sensor class hierarchy, models all actuators. Each Actuator has some processes, modeled in the class Process and defining the functions of the actuators. For example, the Switch has two processes corresponding to the switch on and switch off functions.

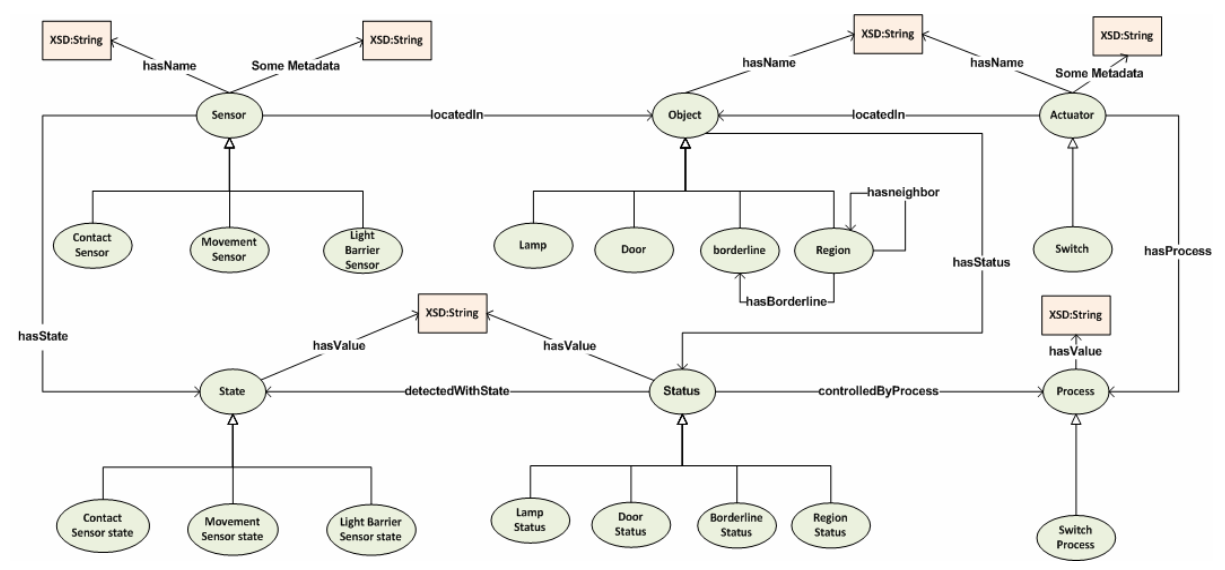

Fig. 4. Illustration of the main concepts in the domain ontology

The Object hierarchy describes the real world entities such as Lamp, Door and Region, which are connected to a sensor or an actuator. The object property locatedIn describes the connection between the Objects and Sensors or Actuators. Each object has several statuses e.g. Door has two statuses: open and closed. Some of these statuses can be detected by Sensor with the special State; the others are controlled by Actuator by using related Process.

This ontology is used as background knowledge by ETALIS engine. Indeed, ETALIS allows using background knowledge in the detection process - any constraint can be easily associated to each situation, which enables a very easy generation of new occupancy situations that should be detected. For example, it is very easy to introduce a new property of a region in an office, like to treat regions that have a window separately from other regions. 


\section{Evaluation}

In order to evaluate the performance of the proposed system we have implemented a test case concerning efficient energy consumption in an office. We have used the FZI Living $\mathrm{Lab}^{8}$ environment for the testing.

The use case is based on simulating occupancy control situations that limit the operation of the lighting system based on the actual use of the space. In other words, if there is a situation, that leads to possibly saving energy, being recognized in a way specified in Section 3.3, the corresponding lighting source should be either dimmed or switched off. In order to make the test realistic we have implemented the set of energy consumption patterns developed for a Building Energy Challenge ${ }^{9}$. Table 1 represents some of those patterns. Note that in order to be realistic we assume that there are negative and positive situations from the energy efficiency point of view, depicted as Penalties and Bonus in Table 1 (the setting has been completely taken from the Building Energy Challenge).

Table 1. Examples of the consumption patterns from the Building Energy Challenge

\begin{tabular}{|l|l|}
\hline \multicolumn{1}{|c|}{ Penalties } & \multicolumn{1}{c|}{ Bonus } \\
\hline $\begin{array}{l}\text { Having a window open while the heating } \\
\text { system is on }\end{array}$ & $\begin{array}{l}\text { Switch off the light each time when } \\
\text { leaving the office }\end{array}$ \\
\hline $\begin{array}{l}\text { Leaving the office at the end of the day } \\
\text { with the computer switched on }\end{array}$ & $\begin{array}{l}\text { Switch off the heating each time when } \\
\text { none is in the office }\end{array}$ \\
\hline $\begin{array}{l}\text { Switch on the artificial light while day } \\
\text { light is sufficient }\end{array}$ & $\begin{array}{l}\text { Switch off the computer when leaving } \\
\text { the office for more than one hour }\end{array}$ \\
\hline $\begin{array}{l}\text { Having a temperature lower than } 26^{\circ} \mathrm{C} \\
\text { with the air conditioning on [12] }\end{array}$ & $\begin{array}{l}\text { Switch off the artificial light while day } \\
\text { light is sufficient }\end{array}$ \\
\hline
\end{tabular}

We have modeled all these patterns using ETALIS language and ontologies as discussed in Section 3. The setting of the sensors was very similar to that presented in Figure 1 (additional sensors for measuring temperature, light intensity and actuators for electric devices have been introduced).

We performed an experiment in order to measure savings in the energy consumption. We have measured the power saving time in the period of one month in an office with five people. We find this setting as a very common one. As already explained, our declarative approach doesn't depend on the number of sensors and the

\footnotetext{
${ }^{8}$ Living Labs is a practical approach to realize open innovation with a regional dimension. By definition, it is "research methodology for sensing, validating and refining complex solutions in real life contexts". It is conceptualized as an innovation platform that brings together and involves a multitude of actors, such as end-users, researchers, industrialists and policy makers. They crucial characteristic is that they are user-centered with an active participation of users within the entire development process. Usually, Living Labs build upon or create a technology platform geared to answer the needs of users in a particular situation.

${ }^{9}$ A contest regarding energy consumption between several office buildings within a company, see: http://www.artist-embedded.org/docs/Events/2010/GREEMBED/0_GREEMBED_Papers/ IntUBE 20- 20GREEMBED.pdf
} 
size of the room. We performed several changes in the layout of the room (position of sensors) but without the need to change the complex event patterns. Therefore, the abstraction provided by our language is correct: interesting situations are defined on the level of objects, independently from the current position of sensors.

Table 2 presents the results from this experiment. In the last column we present the average value of measurements and in the rest of the columns the values for four particular days $\left(1 \mathrm{st}, 10^{\text {th }}, 20^{\text {th }}\right.$ and $30^{\text {th }}$ ) in order to illustrate how theses consumption values varied.

Power saving time represents the time when some electric devices were switched off because of the situation that the corresponding person (related to that device) had left the room.

We are quite satisfied with the general result of the experiment: the proposed approach leads to significant reductions in the energy consumption. We didn't encounter any example of the false positive.

Table 2. The results from the experiment

\begin{tabular}{|l|l|l|l|l|l|}
\hline & $1^{\text {st }}$ day & $10^{\text {th }}$ day & $20^{\text {th }}$ day & $30^{\text {th }}$ day & average \\
\hline $\begin{array}{l}\text { Power } \\
\text { Saving time: }\end{array}$ & $33089 \mathrm{~s}$ & $19548 \mathrm{~s}$ & $58133 \mathrm{~s}$ & $42152 \mathrm{~s}$ & $\mathbf{3 8 2 5 5}$ \\
\hline Total time: & $109199 \mathrm{~s}$ & $103665 \mathrm{~s}$ & $124676 \mathrm{~s}$ & $117021 \mathrm{~s}$ & $\mathbf{1 1 1 3 5 4 s}$ \\
\hline Proportion: & $30.3 \%$ & $18.9 \%$ & $46.6 \%$ & $36 \%$ & $\mathbf{3 4 . 3 \%}$ \\
\hline Error: & 12 & 13 & 18 & 10 & $\mathbf{1 3}$ \\
\hline Total switch: & 67 & 56 & 59 & 56 & $\mathbf{6 2}$ \\
\hline Error rate & $17.9 \%$ & $23.2 \%$ & $30.5 \%$ & $17.9 \%$ & $\mathbf{1 8 . 3 \%}$ \\
\hline
\end{tabular}

The only problem we have faced is the rather huge error rate, whereas an error represents the number of situations that couldn't be detected by using currently deployed patterns (out of scope of the experiment). In the following we give an explanation (i.e. interfere factors) of these situations.

The first interfere factor is the precision of the sensors. In the evaluation we used ELV FS20 sensor systems including FS20 PIRI-2 motion sensor, FS20 IR light barrier sensor, FS20 TFK contact sensor and FS20 ST-3 radio electrical socket. The motion sensor and the light barrier sensor have a minimal send time interval of 8 seconds, which means they can only send a single value every 8 seconds. In the case of a high activity frequency, the sensors can't detect all activities. Furthermore, the sensors can't detect some situations such as two people come into the office together. In this situation the sensors are not able to recognize the number of the people and only one lamp will be switched on. To overcome this, we can use more sensors and the better sensors to increase the precision of the event detection.

The second interfere factor is unanticipated activity in the office. For example, a user forgets to close the door after coming into the office. Then when another user leaves the office, he doesn't need to open the door, which is a necessary event according to the pattern. In this situation, the lamp will also not be switched off. Similarly, a visitor has visited the office, when he leaves the office, one lamp in the 
office will be falsely switched off. This problem can be overcome by installing automatic door closing device and using new sensor technologies (such as RFID) to recognize the identity of the user.

The third interfere factor results from the fact that the pattern definition doesn't match the character of a user. In the pattern we have defined that the movement event and door open event must happen within 5 seconds to trigger the switch off event. If a user is accustomed to do something else costing more than 5 seconds before he opens the door, then his lamp will not be switched off. The problem can be solved by doing some study on the characters of the users before defining the patterns.

Modeling the above mentioned situations will be one of the subjects of the further work.

\section{Related Work}

In this section we only present the related work related to the current lighting control systems. Related work to our approach for complex event processing can be found in [2].

Current lighting and climate control systems often rely on building regulations that define maximum occupancy numbers for maintaining proper lighting and temperatures. However, in many situations, there are rooms that are used infrequently, and may be lighted, heated or cooled needlessly. Having knowledge regarding occupancy and being able to accurately predict usage patterns may allow significant energy-savings.

In [13], the authors reported on the deployment of a wireless camera sensor network for collecting data regarding occupancy in a large multi-function building. They constructed multivariate Gaussian and agent based models for predicting user mobility patterns in buildings.

In [14], the authors identified that the majority of this energy waste occurs during the weekdays, not during the weeknights or over the weekends. They showed that this pattern of energy waste is particularly suited to be controlled by occupancy sensors, which not only prevent runaway operation after typical business hours, but also capture savings during the business day.

An analysis of the impact of the new trends in energy efficient lighting design practices on human comfort and productivity in the modern IT offices is given in [14].

In [15], the authors presented the design and implementation of a presence sensor platform that can be used for accurate occupancy detection at the level of individual offices. The presence sensor is low-cost, wireless, and incrementally deployable within existing buildings.

An examination of different types of buildings and their energy use is given in [16]. The authors discussed opportunities available to improve energy efficient operation through various strategies from lighting to computing.

As a conclusion, there are many approaches for the lighting control, but none of them is using a more declarative approach that would enable an efficient real-time situation detection. 


\section{Conclusions}

In this paper we presented a novel approach for achieving energy efficiency in public buildings (especially sensor-enabled offices) based on the application of intelligent complex event processing and semantic technologies. In the nutshell of the approach is an efficient method for realizing real-time situational awareness that helps in recognizing the situations where a more efficient energy consumption is possible and reaction on those opportunities promptly. Semantics allows a proper contextualization of the sensor data (its abstract interpretation). Complex event processing enables the efficient real-time processing of sensor data and its logic-based nature supports a declarative definition of the situations of interests.

The approach has been implemented using iCEP framework and deployed in the FZI Living Lab environment that supports testing novel technologies with a higher users' involvement. We have performed a case study related to the office occupancy control, that limits the operation of the lighting system based on the actual use of the space. Preliminary evaluation tests have shown very promising results regarding the usability and the efficiency of the approach: the approach is able to abstract from particular patterns to be recognized into general/declarative situations to be reasoned about.

Future work will be related to modeling a more comprehensive set of patterns for representing more complex situations as described in the Evaluation section. Additionally, new tests in the Living Lab have been planned.

\section{Acknowledgments}

Research for this paper was partially financed by EU in the following FP7 projects: ALERT (ICT-258098), PLAY (ICT-258659) and ARtSENSE (ICT-270318).

\section{References}

1. Thirunarayan, K., Henson, C., Sheth, A.: Situation Awareness via Abductive Reasoning for Semantic Sensor Data: A Preliminary Report. In: Proceedings of the 2009 International Symposium on Collaborative Technologies and Systems (CTS 2009), Baltimore, MD, May 18-22 (2009)

2. Anicic, D., Fodor, P., Rudolph, S., Stuehmer, R., Stojanovic, N., Studer, R.: A rule-based language for complex event processing and reasoning. In: Proceedings of the 4th International Conference on Web Reasoning and Rule Systems (RR 2010), pp. 42-57 (2010)

3. Deepak, M.: SNOOP: An Event Specification Language For Active Database Systems. Master Thesis, University of Florida (1991)

4. Carney, D., Cetintemel, U., Cherniack, M., Convey, C., Lee, S., Seidman, G., Stonebraker, M., Tatbul, N., Zdonik, S.: Monitoring streams: a new class of data management applications. In: VLDB 2002: Proceedings of the 28th international conference on Very Large Data Bases. VLDB Endowment (2002)

5. Ray, O.: Nonmonotonic abductive inductive learning. Journal of Applied Logic (2008) 
6. Gutierrez, C., Hurtado, C.A., Vaisman, A.A.: Introducing time into rdf. IEEE Transactions on Knowledge and Data Engineering 19(2), 207-218 (2007)

7. Ryvkina, E., Maskey, A.S., Cherniack, M., Zdonik, S.: Revision processing in a stream processing engine: A high-level design. In: Proc. Int. Conf. on Data Eng (ICDE), Atlanta, GA, USA (2006)

8. Agrawal, J., Diao, Y., Gyllstrom, D., Immerman, N.: Efficient pattern matching over event streams. In: Proceedings of the 28th ACM SIGMOD Conference, pp. 147-160 (2008)

9. Barga, R.S., Goldstein, J., Ali, M.H., Hong, M.: Consistent streaming through time: A vision for event stream processing. In: Proceedings of the 3rd Biennial Conference on Innovative Data Systems Research (CIDR 2007), pp. 363-374 (2007)

10. Arasu, A., Babu, S., Widom, J.: The cql continuous query language: semantic foundations and query execution. VLDB Journal 15(2), 121-142 (2006)

11. Anicic, D., Stojanovic, N.: Expressive logical framework for reasoning about complex events and situations. In: Intelligent Event Processing - AAAI Spring Symposium 2009. Stanford University, California (2009)

12. Recommendation from the French Construction code for construction and housing, http://www. legifrance.gouv.fr/affichCodeArticle.do; jsessionid=87AE72FAE86DC9CF56B8673C1B88F9AD.tpdjo08v_2? cidTexte=LEGITEXT000006074096\&idArticle= LEGIARTI000006896264\&dateTexte $=20090619 \&$ categorieLien $=i d$

13. Erickson, V.L., et al.: Energy Efficient Building Environment Control Strategies Using Real-time Occupancy Measurements. In: Proceedings of the First ACM Workshop on Embedded Sensing Systems for Energy-Efficiency in Buildings, pp. 19-24 (2009)

14. von Neida, B., et al.: An analysis of the energy and cost savings potential of occupancy sensors for commercial lighting systems,

http://www. Irc.rpi.edu/resources/pdf/dorene1.pdf

15. Walawalkar, R., et al.: Effect of Efficient Lighting on Ergonomic Aspects in Modern IT Offices,

http: / / www. walawalkar.com/info/Publications/Papers /

EE\&Ergonomics.pdf

16. Agarwal, Y., et al.: Occupancy-driven energy management for smart building automation. In: Proceedings of the 2nd ACM Workshop on Embedded Sensing Systems for EnergyEfficiency in Building (2010) 